\title{
Combining bioinformatic prediction and assay experiment to identify novel xanthine oxidase inhibitory peptides from Pacific bluefin tuna (Thunnus Orientalis)
}

\author{
Ying $\mathrm{Bu}^{1}$, Fei Wang ${ }^{1}$, Wenhui Zhu*1, Xuepeng $\mathrm{Li}^{1}$ \\ ${ }^{1}$ College of Food Science and Engineering, Bohai University, National\& Local Joint Engineering Research Center of Storage, \\ Processing and Safety Control Technology for Fresh Agricultural and Aquatic Products, 121103, China
}

\begin{abstract}
In this work, we aim to combine bioinformatic prediction with a special experiment to search xanthine oxidase (XOD) inhibitory peptides from myosin of Pacific bluefin tuna (Thunnus Orientalis). The program Peptide Cutter, Peptide Ranker, Peptide Property calculator, Toxin Pred, and Discovery Studio (DS) help us screen the probable sequence. The result indicated that peptide ICRK has the highest inhibition effect and the value of $\mathrm{IC}_{50}$ was $14.18 \mathrm{mg} / \mathrm{mL}$. The $\mathrm{IC}_{50}$ of the other two peptides (FDAK and MMER) were $16.8 \mathrm{mg} / \mathrm{mL}$ and $15.3 \mathrm{mg} / \mathrm{mL}$ respectively. Molecular simulation demonstrated that ICRK interacted with amino acid residues GLU802, PHE914, ALA1079, GLU1261, LYS771, LEU648, THR1010, VAL1011 and SER 876. The possible inhibition mechanism of peptides and enzyme was stated by DS. Peptide ICRK blocked the entrance to the hydrophobic channel and stopped xanthine going into the active site of XOD. MMER and FDAK have the similar mechanism with ICRK. Therefore, ICRK, FDAK and MMER can be considered as nature XOD inhibitory peptides and further utilized.
\end{abstract}

\section{Introduction}

Gout is regarded as a metabolic disease, which leads deposition of urate in the joints and acute bouts of painful inflammatory arthritis. High level of serum uric acid over a long period can cause the symptoms of gout 1 . Xanthine oxidase (XOD) is an enzyme with low specificity, which can catalyze the formation of xanthine and then uric acid from hypoxanthine, and directly catalyze the formation of uric acid from xanthine 2. Inhibition the activity of XOD could decrease the content of uric acid in vivo and relieve the symptoms of gout. Clinic drug allopurinol can effectively control serum uric acid level and has been used extensively. Allopurinol, an analog of purine, is a kind of noncompetitive inhibitor of XOD3. However, serious side-effects vastly restrict its application. Allopurinol could lead hypersensitivity syndrome, impaired liver function, and renal toxicity with some patients 4 . Therefore, it is necessary for us to search for valid and nontoxic XOD inhibitors.

In this paper, we plan to generate and characterize XOD inhibitory peptides from Pacific bluefin tuna (Thunnus Orientalis). Firstly, myosin was hydrolysed with pepsinand trypsin in silico, and the potential activity of myosin-derived XOD inhibitory peptides was evaluated. Subsequently, we conducted molecular docking of XOD inhibitory peptides with XOD ligand. After identifying and synthesizing the XOD inhibitory peptides, the activity of the screened XOD inhibitory peptides will be identified through assay experiment. This work may be helpful for discovering new XOD inhibitory peptides from marine resource.

\section{Materials and methods}

\subsection{Materials and regents}

Bovine milk xanthine oxidase (EC1.17.3.2, 0.5 units/mg protein) and xanthine ( $>98 \%$ ) were bought from Solarbio (Beijing, China) and were stored at $4^{\circ} \mathrm{C}$. Allopurinol was purchased from Shanghai Macklin Biochemical Co., Ltd (Shanghai, China). The peptides used in this study were synthesized by conventional Fmoc solid-phase from Sangon Biotech Co., Ltd. (Shanghai, China) and the purity of peptides were not less than 98\%. Tetrabutylammonium hydroxide, glacial acetic acid, and methylalcohol are chromatographic. Methylalcohol was purchased from Thermo fisher Scientific (Shanghai, China). Tetrabutylammonium hydroxide was purchased from TCL (Shanghai, China). Glacial acetic acid was purchased from FUCHEN Chemical reagents Co. (Tianjin, China). Other reagents were analytical reagents.

Corresponding author's e-mail: zhuwenhui@bhu.edu.cn 


\subsection{Virtual hydrolyze of protein in Pacific bluefin tuna using computerized program}

The myosin was acquired from Pacific bluefin tuna and its sequences can be gotten from NCBI (GenBank:BAL27686.1). Pepsin and trypsin which were mainly nonspecific enzymes in digestive system can disintegrate macromolecule into micromolecule. The sequence of peptides that released from myosin were predicted using the program ExPASy peptide cutter (https://web.expasy.org/peptide_cutter/) 5 .

\subsection{The toxicity and physicochemical properties prediction}

The program ToxinPred was used for predicting toxicity of the selected peptides in silico (http://www.imtech.res.in/raghava/toxinpred/). For screening XOD inhibitory peptides, we used Peptide Ranker to estimate bioactivity of peptides and give a score for peptides. In addition, physicochemical properties like hydrophobicity, molecular weight, isoelectric point $(\mathrm{pI})$ and steric hindrance were also estimated. The solubility of peptides can be predicted by another program Peptide Property Calculator, which can estimate whether peptides have good solubility, and available

at https://www.novoprolabs.com/tools/calc_peptide_proper ty6.

\subsection{The energy evaluation of the protein and peptides complexes}

Molecular docking was performed to screen valid XOD inhibitors using Discovery Studio (v17.2.016349) in silico. The crystal structure of XOD was obtained from Protein Data Bank (Code: 1N5X, a crystal structure of xanthine oxidase-TEI-6720) 7. Peptides were constructed in small molecule window. Firstly, prepared the protein, including removing water molecule in protein and optimizing its structure. Then, using CHARMm force to minimize the peptides energy. CDOOKER is a kind of semi-flexible docking manner based on the energy minimization. The docking coordinates: X 96.7091, Y 55.2721 and Z 40.5282. The radius of the complex was set as 12 . Set up the parameter as Top Hit of 10 and a pose cluster radius of 0.1 .

\subsection{In vitro inhibition activity evaluation of XOD}

The XOD inhibitory activity was performed according to the method of Li8 and measured by the reduction rate of xanthine content in the enzymatic reaction.

\section{Result and Discussion}

\subsection{The XOD inhibitory peptides in myosin}

In the virtual simulation experiment, a total of 240 XOD inhibitory peptides were generated from tripeptides to nonapeptides, including eighty one tripeptides, forty eight tetrapeptides, thirty three pentapeptides, thirty five hexapeptides, twenty one heptapeptides, tenoctapeptides and twelve nonapeptides. We predicted the scores of 240 XOD inhibitory peptides through program peptide ranker to screen active peptides. The higher the score of the peptide, the more likely it is to be bioactive. The peptides sequence with score greater than 0.4 were searched. It can be seen that a total of 30 XOD inhibitory peptides have score over 0.4 , among which NPPNF has the highest score of 0.93 , next was MCR (0.92).

\subsection{Molecule docking of XOD inhibitory peptides with XOD}

In order to find out proper XOD inhibitors, we use molecular docking to acquire the minimization energy between XOD inhibitory peptides and XOD. The result showed that eight of peptides (including LQQEISD, NHHMFV, STHPHF, EEECMF, NPPNF, LMNMK, WLPVY, FMNVK) cannot combine with XOD, which indicated these peptides have no XOD inhibiting effect. According to the interaction energy, we selected six peptides with the lowest interaction energy for further study. Their sequences expressed as ICRK, FDAK, MMER, FDK, QPR and GGK, and their interaction energy ranged from $-81.821 \mathrm{kcal} / \mathrm{mol}$ to -63.0494 $\mathrm{kcal} / \mathrm{mol}$. Low interaction energy value represents strong combination while high represents weak combination 9 . Peptide ICRK has the lowest interaction energy (-81.821 $\mathrm{kcal} / \mathrm{mol}$ ) among the six peptides, so it was most likely to inhibit XOD activity. FDAK and MMER also have proper interaction energy, -78.1075 and -74.1865 $\mathrm{kcal} / \mathrm{mol}$, respectively.

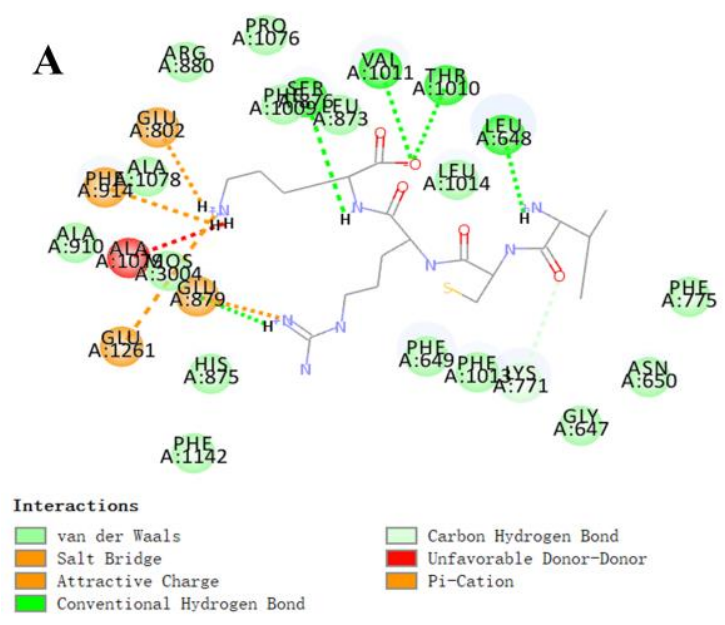



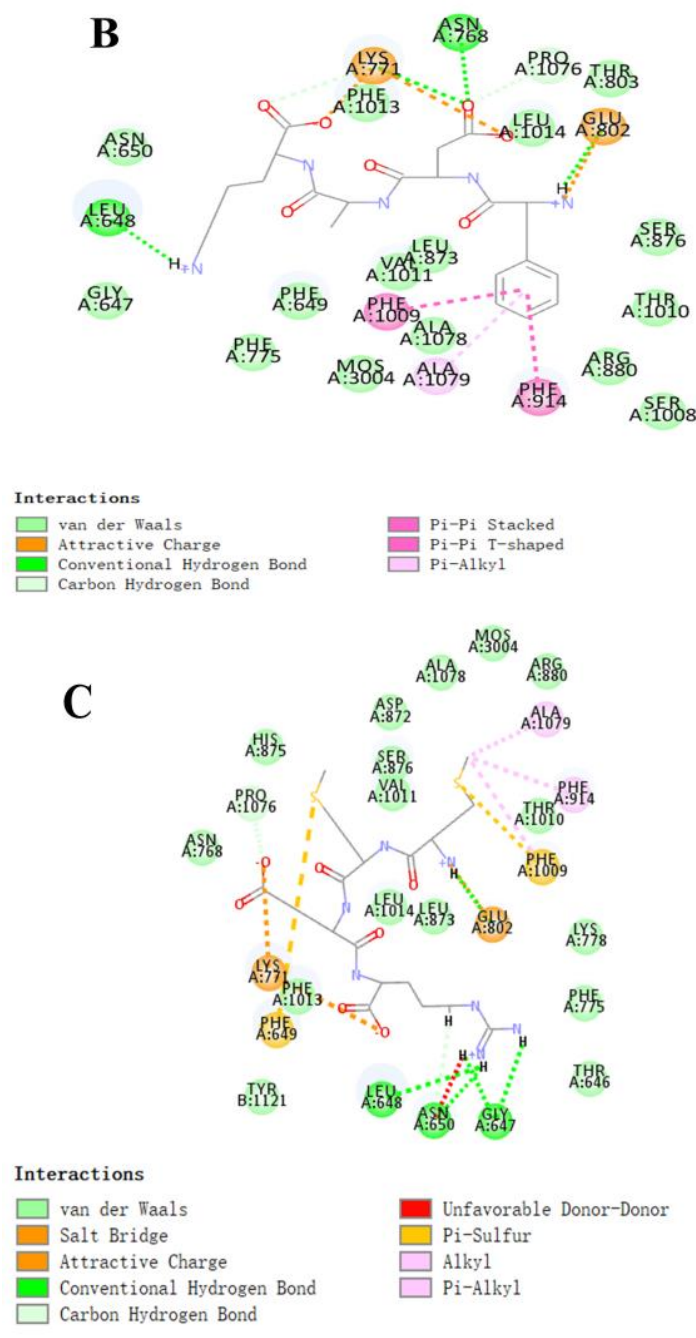

Figure 1 The interaction force between XOD receptor and

$$
\text { ICRK (A), FDAK (B) and MMER (C). }
$$

XOD contain four active sites, among which the active molybdenum and ligand FAD play an important role 10. Xanthine is reduced at the molybdenum center and oxidized via FAD. As shown in Figure 1A, affinity bonds between peptide ICRK and XOD contain conventional hydrogen bond, salt bridge, carbon hydrogen bond and unfavorable donor-donor. ICRK contact with GLU802 through salt bridge; GLU879, GLU1261 and PHE914 through tractive charge; AlA1079 through unfavorable donor-donor; LYS771 through carbon hydrogen bond; SER876, THR1010, VAL1011, LEU648 through conventional hydrogen bond (Figure 1A). There were five hydrogen bonds between XOD with SER876, VAL1011, THR1010, LEU648 and GLU879 in the docking interaction.

FDAK bound to XOD residues LEU648, ASN768 and GLU802 by conventional hydrogen bond, LYS771, GLU802 with attractive charge, PHE1009, PHE914 with pi-pi stack, ALA1079 with pi-alkyl, PRO1076 with carbon hydrogen (Figure 1B). Four hydrogen bonds were formed between XOD with LYS771, ASN768, GLU802 and LEU648 in the docking interaction.
MMER contact with residues PRP1076 and ASN650 by carbon hydrogen bond; LYS771 by attractive charge; GLU802 by salt bridge; PHE649, PHE1009 by pi-sulfur; GLY647, ASN650, LEU648 with conventional hydrogen bond (Figure 1C). There were four hydrogen bonds between XOD with the three residues of LEU648, ASN650 and GLY647.Glu802, Arg880 and GLU1261 were important residues of xanthine. ICRK connected GLU802 and GLU1261 via salt bridge. FDAK connected with the residue GLU802 by Hydrogen bond and attractive charge. MMER and FDAK were also connected GLU802 by Hydrogen bond and attractive charge. They all connected important residues GLU802 with XOD. These bonds strengthened combination between molecules and proteins. All the three peptides contain two hydrophobic amino acids, ICRK contains isoleucine and cysteine, FDAK contains phenylalanine and valine, and MMER contains two methionines. Meanwhile, some hydrophobic amino acids PHE1142, ALA910, ALA1078, PRO1076, PHE1009, PHE775, PHE1013 and PHE649 were around the binding sites, which demonstrated hydrophobic interaction was an important force between peptides and XOD. As figure2 described, there was a hydrophobic pocket next to MOS and formed a path to active site. ICRK went deep into the entrance to MOS (Figure 1B), and prevented xanthine from entering the active center and decreased activity of XOD. This may be the inhibition mechanism of ICRK and this result was similar with Li's study11. As for peptide FDAK and MMER, their inhibition mechanism are similar as ICRK.

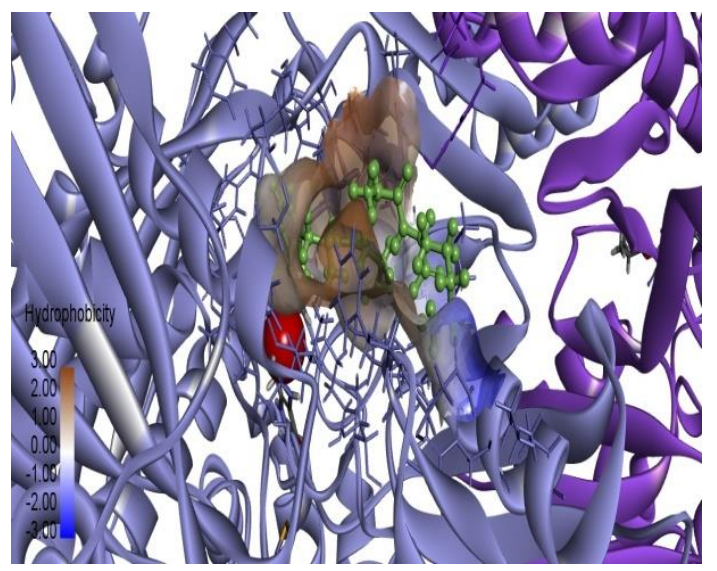

Figure 2 The XOD inbibitory mechanism of ICRK.

\subsection{In silico prediction of toxicity and property of XOD inhibitory peptides}

All the six XOD inhibitory peptides were nontoxic with good solubility. Solubility play an important role in the reaction. Generally, good solubility is conducive to absorption and treatment, while poor solubility will restrict bioavailability and clinical translations 12 .

Some identified peptides have been collected and listed in Table 1. The three identfied peptides ICRK, MMER, FDAK of this study have not been reported. 
Hydrophobicity can influence the bioaccessibility of peptides in the gastrointestinal13. Li et al. found the hydrophobic peptide WML entered the active site of XOD more easily than the hydrophilic peptide PGACSN11. The hydrophobicity values in our prediction were minus, which implied these peptides were hydrophilic, and these peptides connected protein by hydrogen-bonding and electrostatic interactions. The $\mathrm{pI}$ is the $\mathrm{pH}$ where the net charge on the molecular ensemble of peptides and proteins is zero14. Referring to this result, we can select proper $\mathrm{pH}$ in this experiment.

Table 1 The comparision of identified XOD inhibitory peptides.

\begin{tabular}{|c|c|c|c|c|c|}
\hline Peptide & Origin & $\mathrm{IC}_{50}$ & Peptide & Origin & $\mathrm{IC}_{50}$ \\
\hline WPPKN & Walnut11 & $\begin{array}{c}17.75 \\
\mathrm{mg} / \mathrm{mL}\end{array}$ & WDD & & $2.41 \mathrm{mM}$ \\
\hline ADIYTE & & $19.01 \mathrm{mg} / \mathrm{mL}$ & $\mathrm{HCPF}$ & & $\begin{array}{l}15.07 \\
\mathrm{mM}\end{array}$ \\
\hline AAAAGAKAR & Oryza Sativa15 & -- & WDQW & & $0.95 \mathrm{mM}$ \\
\hline $\mathrm{FCH}$ & Pleurotusostreatus 16 & $\begin{array}{r}0.90 \\
\mathrm{mg} / \mathrm{mL}\end{array}$ & PPKNW & & $2.21 \mathrm{mM}$ \\
\hline FH & Tuna1717] & $25.7 \mathrm{mM}$ & WSREEQE & Walnut20 & $1.88 \mathrm{mM}$ \\
\hline WV & Milk protein 18 & $1.30 \mathrm{mM}$ & VWPP & & $1.86 \mathrm{mM}$ \\
\hline WML & & -- & FRRY & & $4.72 \mathrm{mM}$ \\
\hline PGACSN & Bonito19 & -- & VPPW & & $1.61 \mathrm{mM}$ \\
\hline AMPF & & -- & TEPPR & & $5.45 \mathrm{mM}$ \\
\hline FGVG & & -- & FFKG & & $7.26 \mathrm{mM}$ \\
\hline ICRK & & $7.23 \mathrm{mg} / \mathrm{mL}$ & FTPRF & & $9.61 \mathrm{mM}$ \\
\hline FDAK & Pacific bluefin tuna in & $14.18 \mathrm{mg} / \mathrm{mL}$ & $\mathrm{CFPH}$ & & $13.57 \mathrm{mM}$ \\
\hline MMER & this study & $16.30 \mathrm{mg} / \mathrm{mL}$ & ACECD & Bonito21 & $\begin{array}{c}7.23 \\
\mathrm{mg} / \mathrm{mL} \\
\end{array}$ \\
\hline
\end{tabular}

\subsection{In vitro XOD activity inhibition assay}

To further verify XOD inhibitory effect of the identified peptides, we synthesized the three peptides and conducted assaying experiment. $\mathrm{IC}_{50}$ of identified peptides in previous studies and this study were listed in table 1. The $\mathrm{IC}_{50}$ of allopurinol was $14.6 \mu \mathrm{g} / \mathrm{mL}$, and $\mathrm{IC}_{50}$ of peptides ICRK, FDAK and MMER were 14.18 $\mathrm{mg} / \mathrm{mL}, 16.80 \mathrm{mg} / \mathrm{mL}$ and $15.30 \mathrm{mg} / \mathrm{mL}$, respectively. $\mathrm{IC}_{50}$ of peptide ACEC which derived from bonito was $7.23 \mathrm{mg} / \mathrm{mL} 21$, FH derived from tuna was $25.7 \mathrm{mM} 17$. $\mathrm{IC}_{50}$ of peptides WPPKN and ADIYTE derived from walnut were $17.75 \mathrm{mg} / \mathrm{mL}$ and $19.01 \mathrm{mg} / \mathrm{mL}$, respectively 11 . The results showed that the three peptides had a certain inhibitory effect on XOD, and can be considered as candidates of gout suppressant.

\section{Conclusion}

Through our work, combining bioinformatic prediction with assay experiment, we discovered three novel XOD inhibition peptides and explained their inhibition mechanism. The peptides ICRK, MMER and FDAK had a certain inhibition effect and they can be used as potential anti-hypouricemic agents to apply. Molecular docking can be thought as an effective and convenient way for screening effective components.

\section{Acknowledgements}

This study was supported by the National Key Research and Development Program (2018YFD0400603) and LiaoNing Revitalization Talents Program (XLYC1807133).

\section{References}

1. Tan,P.K., Liu, S., Gunic, E., Miner, J.N. (2017) Di scovery and characterization of verinurad, a potent a nd specific inhibitor of URAT1 for the treatment of hyperuricemia and gout. Sci RepUK.,7 (1): 66.

2. Li, Q.Y., Shi, C.C., Wang,M., Zhou,M., Liang,M., Z hang,T., Yuan,E.D., Wang,Z., Yao,M. J.,Ren,J.Y. (2 019a)Tryptophan residue enhances in vitro walnut $p$ roteinderived peptides exerting xanthine oxidase inh ibition and antioxidant activities. JFunct Foods., 53: 276-285.

3. Malik, N., Dhiman, P., Khatkar, A. (2019) In silico design and synthesis of hesperitin derivatives as new xanthine oxidase inhibitors. BMC Chemistry., 13(53).DOI:10.1186/s13065-019-0571-1.

4. Wang, Y.J., Zhang, G.W., Pan, J.H., Gong, D.M.(2015) Novel insights into the inhibitory mechanism of kaempferol on xanthine oxidase. JArg Food Chem., 63(2):526-534.

5. Moayedi, A., Mora, L., Aristoy, M.C., Safari, M., Hashemi, M., Toldrá, F. (2018) Peptidomic analysis of antioxidant and ACE-inhibitory peptides obtained 
from tomato waste proteins fermented using Bacillus subtilis. Food Chem.,250:180-187.

6. Gangopadhyay, N., Wynne, K., O’Connor, P., Gallagher, E., Brunton, N.P., Rai, D.K , Hayes, M. (2016)In silico and in vitro analyses of the angiotensin-1 converting enzyme inhibitory activity of hydrolysates generated from crude barley (Hordeum vulgare) protein concentrates. Food Chem.,203:367-374.

7. Okamoto, K., Eger, B.T., Nishino, T., Kondo, S., Pai, E.F., Nishino, T. (2003) An extremely potent inhibitor of xanthine oxidoreductase: crystal structure of the enzyme-inhibitor complex and mechanism of inhibition. J Biol Chem., 278(3):1848-1855.

8. Li, T.T., Ren, L.K., Wang, D.F., Song, M.J., Li, Q.Y., Li, J.R. (2019b) Optimization of extraction conditions and determination of purine content in marine fish during boiling. PeerJ., 7:e6690.DOI: $10.7717 /$ peerj.6690.

9. Yu, Z.P., Wu, S.J., Zhao, W.Z., Ding, L., David S., Chen, F., Li, J.R., Liu, J.B. (2017) Identification and molecular mechanism of a novel myosinderived ACE inhibitory peptide. Food Funct., 9: 364-370.

10. Cao,H.N., Pauff James, M., Russ, H. (2010)Substrate orientation and catalytic specificity in the action of xanthine oxidase. $\mathrm{J}$ Biol Chem.,285(36):28044-28053

11. Li, Q.Y., Kang, X.Y., Shi, C.C., Li,Y.J., Majumder, K., Ning,Z.X.,Ren, J.Y.(2018b) Moderation of hyperuricemicrats via consuming walnut protein hydrolysates diet and identification of new antihyperuricemicpeptides. Food Funct., 9,107-116.

12. Chen, H.B., Khemtong, C., Yang, X.L., Chang, X.L., Gao, J.M. (2011) Nanonization strategies for poorly water-soluble drugs. Drug Discov Today., 16(78):354-360.

13. Acquah, C., Stefano,E.D., Udenigwe, C.C. (2018) Role of hydrophobicity in food peptide functionality and bioactivity. J Food Bioact.,4:88-98.

14. Bjerrum, E. J., Jensen, J.H., Tolborg, J.L. (2017) pI Calculax: Improved prediction of isoelectric point for modified peptides. J Chem Inf Model., 57 (8):1723-1727.

15. Liu, N. X., Wang, Y., Yan, M.F., Bian, W.X., Zeng, L., Yin, S.G., Xiong, Z.Q., Hu, Y., Wang, S.Y., Meng, B.L., Sun, J., Yang, X.W. (2019). A new rice-derived short peptide potently alleviated hyperuricemia induced by potassium oxonate in rats. J Arg Food Chem., 67(1):220-228.

16. Jang, I.T., Hyun, S.H., Shin, J.W., Lee, Y.H., Ji, J.H., Lee, J.S.(2014) Characterization of an antigout xanthine oxidase inhibitor from pleurotusostreatus. Mycobiology., 42(3):296-300.

17. He, W.W., Su, G.W., Sun-Waterhouse, D.X., Geoffrey, I.N., Zhao, M.M., Liu, Y.(2019) In vivo anti-hyperuricemic and xanthine oxidase inhibitory properties of tuna protein hydrolysates and its isolated fractions. Food Chem., 453-461.

18. Nongonierma, A.B., Fitzgerald, R.G. (2012) Tryptophan-containing milk protein-derived dipeptides inhibit xanthine oxidase. Peptides, 37(2):263-272.

19. Li,Y. J., Kang, X. Y., Li, Q. Y., Shi,C. C., Lian, Y. Y., Yuan, E. D., Zhou, M. and Ren, J. Y. 2018c.Anti-hyperuricemic peptides derived from bonito hydrolysates based on in vivo hyperuricemic model and in vitro xanthine oxidase inhibitory activity. Peptides.107: 45-53.

20. Li, Q. Y. (2018c) Study on the structure-activity mechanism of targeting inhibition of xanthine oxidase by uric acid-lowering peptides derived from walnut. [dissertation]. China: South China University of Technology.

21. Zou, L. (2019) Enzymatic preparation, functional evaluation of xanthine oxidase inhibitory peptides from skipiack tuna. [dissertation]. China: Zhejiang University. 